\title{
Acoustic Modes Transformation upon Reflection in Tellurium Dioxide Crystal
}

\begin{abstract}
N.V. POLIKARPOVA* AND V.B. Voloshinov
Faculty of Physics, Lomonosov Moscow State University, GSP-2, Leninskiye Gory, 119991 Moscow, Russia

Generation of acoustic shear waves in crystals is one of the main problem of acousto-optics. Launching of the shear waves is much more difficult than the longitudinal modes because of difficulties related to acoustic contact and also because of problems arising from matching of acoustic and electric parameters of piezoelectric transducer. We obtained the transverse waves as a result of longitudinal-shear modes reflection in a crystal. Crystals having the required effect should possess a strong anisotropy of their elastic properties to realize the effective acoustic mode transformation. In this paper, one of the important cases of the acoustic waves reflection is investigated theoretically and experimentally. The research was carried out in the crystal of paratellurite.
\end{abstract}

DOI: 10.12693/APhysPolA.127.96

PACS: 78.20.Hp, 42.79.Jq, 43.35.Sx, 43.35.+d

\section{Introduction}

Tellurium dioxide $\mathrm{TeO}_{2}$ (paratellurite) crystals are widely used in modern acousto-optic applications as the media of light and sound interaction [1-7]. This uniaxial crystal has gained the increasing popularity due to its unique combination of elastic and photoelastic properties [8-10]. For particular directions of acoustic propagation, the acousto-optic effect is exceptionally strong due to the considerably low phase velocity value $[1,2]$. The effect is the strongest for a specific polarization of the acoustic mode, while a direct generation of the particular mode is technically challenging.

In paratellurite a shear elastic wave propagating close to the [110] direction is most attractive for the applications. The phase velocity for this mode is quite low, $V=616 \mathrm{~m} / \mathrm{s}$. Efficiency of light and sound interaction is determined by the acousto-optic figure of merit $M_{2}$, which is inversely proportional to the third power of the phase velocity value $[1,4,6,7]$. It is known, nevertheless, that there are technical issues related to generation of the shear waves in paratellurite $[6,7]$. Since the piezoelectric transducer is resonant, its fundamental frequency is related to the width of the piezoelectric plate $d=V / 2 f$, where $V$ - sound velocity and $f$ - frequency. The higher the ultrasound frequency, the thinner is the transducer plate and in turn the larger the static capacity $c=\varepsilon S / 4 \pi d$, where $\varepsilon$ - permittivity, $S$ - square of the plate. This capacity usually determines technical complications in electric matching of the transducer and a highfrequency generator. Additional difficulties arise from the difference of acoustic impedances of the transducer and the $\mathrm{TeO}_{2}$ crystal. For example, acoustic wave transfer through a contact between $\mathrm{LiNbO}_{3}$ and $\mathrm{TeO}_{2}$ is inefficient due to the difference in the acoustic impedances

${ }^{*}$ corresponding author; e-mail: polikarp@phys.msu.ru by 5 times [11]. The above complications may be avoided if one induces longitudinal acoustic (L) waves, which are subsequently transformed into shear (S) waves upon the reflection from a crystal-vacuum boundary.

\section{Reflection of plane acoustic waves at crystal-vacuum boundary}

Figure 1 shows general view of the used acousto-optic cell and schematics of transformation of the pure longitudinal wave $\mathrm{L}$ to the slow quasi-shear wave (QSS). As found, the reflection at the boundary results in as much as three waves (shown in Fig. 1): quasilongitudinal (QL) wave and two quasi-shear waves. One of the waves is slow QSS while the other one is fast quasi-shear wave (QFS) [12]. Therefore part of the energy of the initial wave $(\mathrm{L})$ is transformed to the modes QL and QFS, which are of no use for the majority applications.

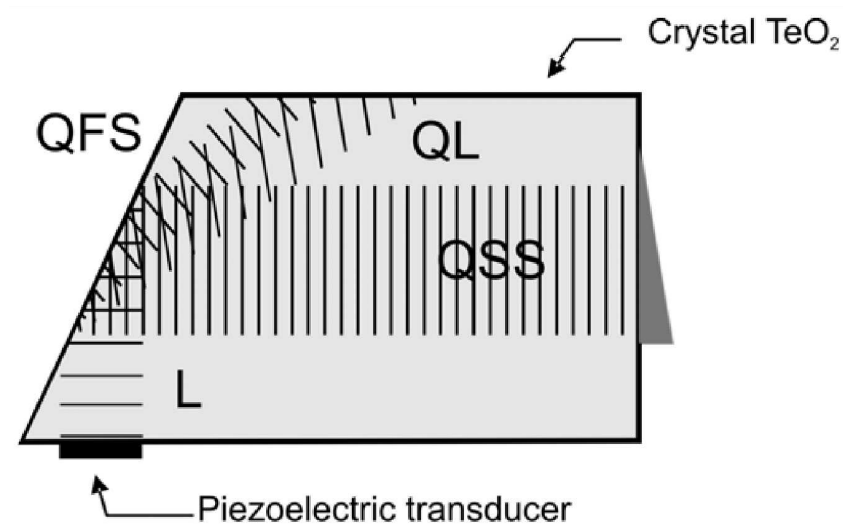

Fig. 1. Acoustic modes transformation scheme at acoustic reflection.

We consider the case when the $\mathrm{L}$ wave is generated along $[1 \overline{1} 0]$ direction and the (QSS) wave travels in $(\overline{1} 10)$ 
plane at an angle $\alpha$ to the [110] axis (Fig. 2). The L wave is generated without any acoustic walk-off while the required transducer is thicker and therefore it possesses a smaller static capacity. We showed that a proper inclination of the crystal boundary relative to [100] and [001] provides an efficient transformation of the major part of energy in the L wave to QSS wave $[12,13]$.

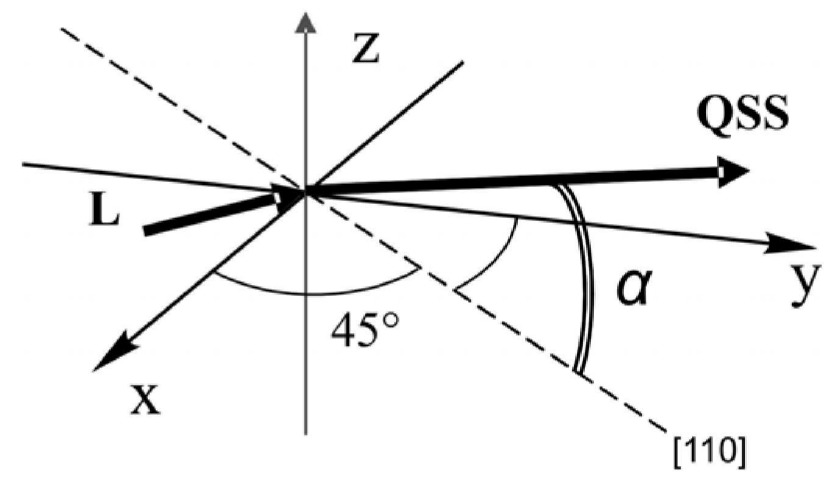

Fig. 2. L into QSS acoustic wave transformation out of the basic plane.

Results of numerical calculations related to the energy reflection coefficient $R$ as a function of the angle $\alpha$ for the three reflected acoustic waves are shown in Fig. 3 [14]. One can see that the transformation efficiency grows with the increase of $\alpha$ and comes close to $100 \%$ when $\alpha=90^{\circ}$. The experimentally obtained value of the reflection coefficient is somewhat below the theoretical estimate due to scattering of acoustic energy on the imperfect crystal boundary, absorption of ultrasound and divergence of the acoustic beam.

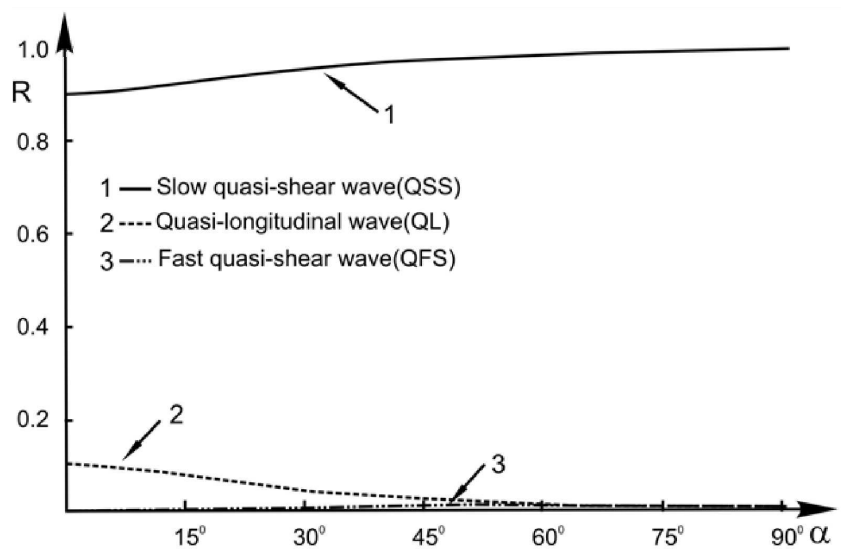

Fig. 3. Energy reflection coefficient $R$ versus the angle $\alpha$ between the reflected wave and direction [110].

It is known that acousto-optic applications are usually based on the waves propagating at the $\alpha$ angle included in the range $0<\alpha \leq \alpha_{\text {cr }}[1,6]$, where the critical angle is $\alpha_{\mathrm{cr}}=18^{\circ}$ in paratellurite. Calculations show that at the critical angle $\alpha=\alpha_{\mathrm{cr}}$ the energy reflection coefficient for the QSS is equal to 93\%. As proved in Fig. 3 the QFS mode may practically be neglected for the angles $\alpha$ below $50^{\circ}$.

\section{Experimental setup and results}

Ratio of incident and reflected acoustic wave intensities was measured in the experiment in order to check the validity of the theoretical predictions. Traditional acousto-optic visualization technique was also employed $[2,12,15-17]$ to control the waves in the paratellurite acousto-optic cell (Fig. 4). The piezoelectric transducer was located on top of the left side of the crystal. The tilt of the upper crystal facet was rather small taking into account the large difference between the velocities of the longitudinal and the shear acoustic modes. The longitudinal wave $\mathrm{L}$ was transformed into the slow quasi-shear wave (QSS) at the reflection and then propagated vertically down. To the left of the crystal, one can see in the picture the electrical matching network.

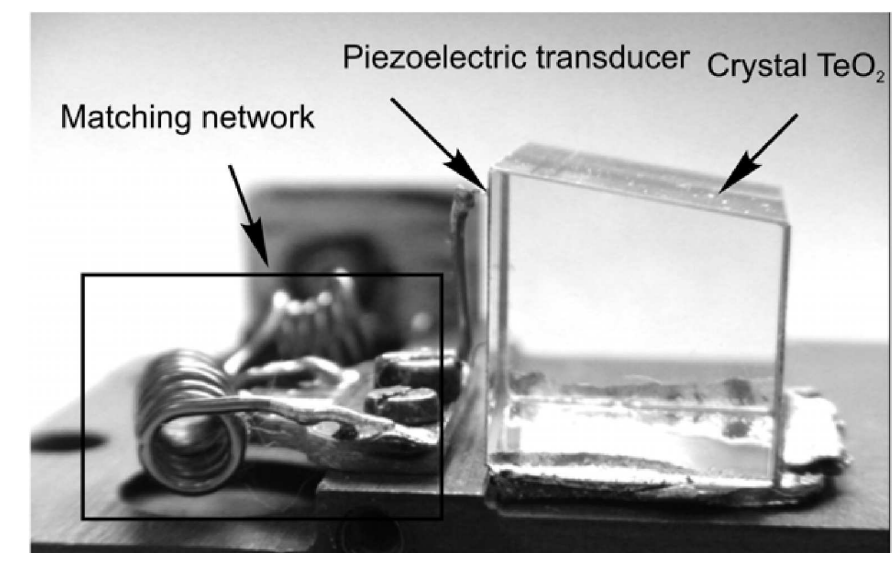

Fig. 4. Acousto-optic cell on basis of tellurium dioxide crystal.

The cell was specially cut with the purpose to direct the wave vector of the reflected slow quasi-shear wave at the angle $\alpha=7^{\circ}$ to the [110] direction in the (110) plane. The frequency of ultrasound was equal to $f=92 \mathrm{MHz}$. Intensity of the diffracted light provided by the two reflected, i.e., the QL and the QSS waves was measured by acousto-optic methods. The ratio of diffracted waves intensities was then recalculated into the ratio of intensities of the corresponding acoustic waves. In the calculation, we used known values of the acousto-optic figure of merit for the two acoustic modes. The resulting ratio for intensities of the QSS and the QL modes was equal to $P_{\mathrm{QS}} / P_{\mathrm{QL}}=6.7 \pm 1.0$, while the theory predicted $P_{\mathrm{QS}} / P_{\mathrm{QL}}=9.0$. According to the theoretical expectation, the QFS was not observed in the experiment at all. Therefore the experimental result confirmed results of calculations of the reflection coefficient. It means that generation of the slow shear acoustic wave by mode transformation in paratellurite is really efficient and may be recommended for usage in new acoustic devices [10, 18]. 


\section{Conclusions}

Transformation of the longitudinal acoustic wave in paratellurite into the slow-shear wave is an efficient technique for generating the slow-shear wave along the direction [110] or close to it. Energy reflection coefficient for such transformation continuously grows from $90 \%$ to $100 \%$ when direction of sound propagation changes from [110] axis to [001] axis in the (110) plane. As found, main losses accompanying the modes transformation are related to the quasi-longitudinal mode, which takes up to $10 \%$ of the incident elastic energy while intensity of the fast quasi-shear reflected wave is negligible. Principal conclusions confirming efficiency of the method were proved experimentally in acousto-optic cell based on paratellurite crystal. Consequently, the transformation of acoustic modes may be recommended for development of new modifications of acousto-optic modulators, deflectors and filters based on the slow shear acoustic waves in paratellurite crystal.

\section{Acknowledgments}

The work is supported by the Russian Science Foundation grant 14-22-00042.

\section{References}

[1] V.I. Balakshy, V.N. Parygin, L.E. Chirkov, Physical Principles of Acousto-Optics, Radio and Svyaz, Moscow 1985 (in Russian).

[2] V.B. Voloshinov, N.V. Polikarpova, Appl. Opt. 48, C55 (2009).
[3] A. Yariv, P. Yeh, Optical Waves in Crystals, Wiley, New York 1984

[4] E. Dieulesaint, D. Royer, Elastic Waves in Solids, Wiley, New York 1981.

[5] B.A. Auld, Acoustic Fields and Waves in Solids, Robert Krieger, New York 1990.

[6] A. Goutzoulis, D. Pape, Design and Fabrication of Acousto-Optic Devices, Marcel Dekker, New York 1994.

[7] J. Xu, R. Stroud, Acousto-Optic Devices: Principles, Design, and Applications, Wiley, New York 1992.

[8] V.A. Burov, V.B. Voloshinov, K.V. Dmitriev, N.V. Polikarpova, Phys.-Usp. 54, 1165 (2011).

[9] N.F. Declercq, N.V. Polikarpova, V.B. Voloshinov, O. Leroy, J. Degrieck, Ultrasonics 44, 833 (2006).

[10] V.B. Voloshinov, Ultrasonics 31, 333 (1993).

[11] V.B. Voloshinov, N.V. Polikarpova, Acta Acust.Acustica 89, 930 (2003).

[12] M.J.P. Musgrave, Geophys. J.R. Astron. Soc. 3, 406 (1960).

[13] V.B. Voloshinov, O.Yu. Makarov, N.V. Polikarpova, Tech. Phys. Lett. 31, 352 (2005).

[14] N.V. Polikarpova, V.B. Voloshinov, Proc. SPIE 5828, 25 (2004).

[15] V.B. Voloshinov, N.V. Polikarpova, N.F. Declercq, J. Acoust. Soc. Am. 125, 772 (2009).

[16] V.B. Voloshinov, N.V. Polikarpova, V.G. Mogaev, Acoust. Phys. 52, 245 (2006).

[17] N.V. Polikarpova, V.B. Voloshinov, Proc. SPIE 5953, 0C1 (2005).

[18] E.A. Djakonov, V.B. Voloshinov, N.V. Polikarpova, Acoust. Phys. 58, 107 (2012). 